Tyndallphänomen richt und sind unbegrenzte Zeit balthar. Thre Leitfăhigkeit verändert sich nicht, es tritt also keine nachträgliche Bildung kolloider Kieselsäure dur ch Hydrolyse ein. Diese Tatsache deuret darauf hin, dab die entstandene
Verbindung kein einfaches Silikat, sondern eine komplex Verbindung darstellt.

Ueber Untersuchungen, die sich mit dieser Frage befassen, wird demnächst gesondert berichtet werden.

\title{
Ueber das Gleichgewicht Gelatine-Saizsäure.
}

\author{
Von Robert Wintgen (Göttingen) und Karl Krüger (Bonn).
}

(Eingegangen am 27, Oktober 1930.)

Die Fähigkeit der Gelatine und der Eiweifkörper im allgemeinen, Saizsäure zu binden, ist bereits häufig untersucht worden. Von neueren Arbeiten, die sich mit diesem Gegenstand beschäftigen, seien die Verôffentlichungen Paul i's und seimer Schüler (s.u.), die von Procter (s. u.) and Jacques Loebi) genannt. Wo.Paulia hat tie Etgebnisse seiner diesbezuglichen umfangreichen und bedeutsamen Untersuchungen in der kürzlich erschienenen ersten Hälfte seiner Kolloidchemie der Eiweißhöryer zusammengefabt. Eine Zusammenstellung der älteren Literatur finder sich bei R. Haber ${ }^{3}$ ) und T. B. Robert . son\%. Da zur Untersuchung der Bindung von Elektrolyten an Eiweißkörper die gewöhnlichen analytischen Hilfsmittel wegen Storung des Gleichgewichts in der Regel nicht anwendbar sind, werden neuerdings hauptsächlich physikalisch-chemische Methoden zur Bestimmung der ungebundenen Salzsăure bzw. der Konzentration der freien Wasserstoffionen angewandt. Von diesen Methoden verdient die heute allgemein übliche Methode der Gasketten zweifellos vor alien andern den Vorzug. Wir haben bei schon einige Jahre zurückliegenden, anfänglich mit einem andern Ziel unternommenen Untersuchungen, über die der eine $\left.{ }^{5}\right)$ von uns seinerzeit. kurz berichtete, uns der Methylazetatkatalyse bedient. (F. A. Hoffmann $n^{6}$ und $O$. Cohnheim maben die

1) Jacques l.oeb, Joum. of Gen. Physlol. 1, 237, 363 (1919).

2) Wo. Pa 1 i, Kolloidchemie der Eiweißkorper, 1. Hälte (Dresden u. Leipzig 1920).

3) R. Hober, Physikalische Chemie der Zelle und der Gewebe, 4. Aufl. (Leipzig 1. Berlin 1914)

*) T. B. Robertson, Die physikalische Chemie der Proteine (Dresden u. Leipzig 1912).

5) R. Wintgen, Ueber das Gleichgewicht GeIatine-Salzsäure. (SB, d. Niederrhein. Ges. fïr Natur und Heilkunde in Bonn, Chemische Abteflung 1915; Collegium 1916, 201.)

6) F A. Hoffmann, Zentralbl. 1 klin. Medizin 1889,773, u. 1890,521 .

7) O.Cohnheim, Zeirschr. f. Biol 39,489 (1896).
Wasserstoffionenkonzentration in Saizsäure-Gelatinegemischen mit Hilfe der Katalyse der Robrzuckerinversion.)

Die Gelatine (Marke Non plus ultra) stammte aus den Deutschen Gelatinewerken A. - G. in Hochstam Main. Zur Bestimmung des Wassergelialtes der luftrocknen Gelatine wurde sie bei $110^{\circ}$ bis zur Gewichtskonstanz getrocknet. Wasserverlust in Pron. 17,27 und 17,23, im Mittel 17,25. Die folgenden Angaben beziehen sich der Einfachheit halber, wenn nicht ausdrïcklich anders bemerkt, stets auf lufttrockne Gelatine. Der Aschengehait betruy 1,66 Proz. Die Gelatine seagierte schwach sauer, $1 \mathrm{~g}$ in $\mathrm{CO}_{2}$. freiem Wasser gelöst verbrauchte mit Phenolphthalein als Indikator (Umschlag unscharf) 3,$34 ; 3,66 ; 3,32 ; 3,69$, im Mittel $3,51 \mathrm{~cm}$ $0,05 n \mathrm{NaOH}$. Diese Azidität, die natürlich bei den Titrationen der spăteren Messungen mit zum Ausdrick kommt, ruft selbst keine Beschleunigung der Esterverseifung hervor, wie aus folgenden Vergleichsversuchen ersichtlich. 2 con einer fünfprozentigen bei $25,0^{\circ}$ im Thermostaten stehenden Methylazeiallösung verbriuchten nach

$\begin{array}{ccccc}0 & 23 & 286 & 630 & 1540 \text { Stunden } \\ 0,00 & 0,01 & 0,82 & 3,14 & 13,51 \quad \mathrm{~cm}\end{array}$

$0,05 \mathrm{n} \mathrm{Na} \mathrm{OH} .2 \mathrm{ccm}$ derselben Lósung, aber unter Zusatz von 2 Proz. Gelatine verbrauchten untèr denselben Bedingungen nach

$\begin{array}{ccccc}0 & 23 & 286 & 630 & 1540 \text { Stunden } \\ 0,15 & 0,15 & 0,19 & 0,28 & 0,41 \mathrm{ccm}\end{array}$

0,05 n NaOH. - Der Gelatinezusatz scheint also im Gegenteil bemerkenswerter Weise eine beträchtliche Verlangsamung der Reaktions. geschwindigkeit hervorzurufen.

Die Versuchsanorduung für unsere Messungen war folgende ${ }^{3}$ ):

In einem $100 \mathrm{ccm}$ fassenden Meßkölhchen wurden $0 ; 0,1 ; 0,2 ; 0,5 ; 1 ; 2 ; 3 ; 4 ; 5$ und

8) Ostwald - Luther, Hand- und Hilfsbuch zur Ausführung physiko-chemischer Messungen, 3. Aufl. (Leipzip 1900), 524. 
6g. Gelatine in etwa $30 \mathrm{ccm}$ Wasser bei $70^{\circ}$ gelost. Nach dem Abkühlen im Thermostaten erhielt jede der Losungen eimen Zusatz von $50 \mathrm{ccm}$ Salzsäure $(0,1 \mathrm{bzw} .0,2 \mathrm{n})$ und $5 \mathrm{~cm}$ Methylazetat. Nach dem Auffullen mit Wasser von $25^{\circ}$ auf $100 \mathrm{ccm}$ und gutem Durchsehütteln wurde von der Mischung in ausgedämpfte 50 ccon fassende Erlenmeyerkölbchen aus Jenaer Glas gefült, die mit Bleiblech beschwert und numeriert waren und bis zum Rand in dem Wasser eines grofen, genan auf $25,0^{\circ}$ eingestellten Thermostaten standen.

Von Zeit zu Zeit wurden mit einer Pipetre $2 \mathrm{ccm}$ des Reaktionsgemisches den Fläschchen entnonmen, die Pipette außen mit Filtrierpapier abgewischt und der Inhalt in ein Becherglas entleert. Beim Entleeren der Pipette in der gewöhnhchen Weise wärde man infolge der verschieden starken Benetzung der Pipetteuwand, die durch die wechselnde Zähigkeit der einzeinen Gelatinelösungen bedingt ist, Fehler erhalten. Um nun bei allen Messungen stets den ganzen lnhalt der Pipette zu titrieren, wurde das aus det Pipette ausgelaufene Reaktionsgemisch mit Natronlauge (Indikator Phenolphthalein) zunächst auf schwach rot titriert, bierauf wieder in die Pipette aufgesaugt und nach dem abermaligen Auslaufen sorgfaltig zu Ende titriert: Die Zeitintervalle $t$ (in Minuten) rechnen von dem Augenblick, in dem der erste Tropfen Methylazetat mit der salzsäurehaltigen Gelatine in Berührung kam, bis. zu dem Beginn der einzeinen Titrationen, $d \cdot b$. bis zu dem Augenblick, in dem der erste Tropfen Lauge in die $z u$ titrierende Lösung fält.

Der Anifangswert $A_{0}$ des Reaktionsverlaufes, bei der alse noch kein Methylazetat verseift ist, wurde, utn Beobachtungsfehler auszuschalten, der Einfachheit hatber in einem besonderen Versuch ohne Zusatz von Methylazetat für alle Messungen festgelegt: In einem $100 \mathrm{ccm}$ Kölbchen wrden $50 \mathrm{ccm} n$-Saizsăure au $100 \mathrm{ccm}$ verdünnt. $2 \mathrm{ccm}$ des Gemisches verbrauchten bei der Titration nach dem oben beschriehenen" Verfahren $20,20 \mathrm{ccm}$ einer $0,05 \mathrm{n} \mathrm{NaOH}$. Hieraus berechnet sich der Anfangswert für die Messungen mit 0,05 und $0,10 \mathrm{n}$ Salzsaure zu 2,02 und 4,04 cerr. Die Bestimmung des Endwertes $A_{\infty}$, der bei den konzentrierten Gelutinelösungen erst nach Monaten erreichbar ist, geschat ebenfalls in einem besonderen Versuche. Zu diesess Zweck wurde eine cntsprechende Mischung ohne Gelatinezusatz angesetzt und nach melurtingigam Stehen in Thermostaten titriert.
Tabelle'l.

$\left.\begin{array}{cc}50 \mathrm{ccm} & \begin{array}{l}1,00 \mathrm{n} \mathrm{HCl} \\ 5\end{array} \\ \text { Methylazetat }\end{array}\right\}$ in $100 \mathrm{ccm}$ Wasser :

\begin{tabular}{l|c|c}
\hline \begin{tabular}{c|c}
$25,0^{\circ}$ \\
Zeit
\end{tabular} & $0,05 \mathrm{n} \mathrm{Na} \mathrm{OH}$ & $0,05 \mathrm{n} \mathrm{NaOH}$ \\
\hline 1. VI. & & II \\
5. VI. & $44,47 \mathrm{ccm}$ & $44,57 \mathrm{ccm}$ \\
6. VI. & $44,56 \mathrm{ccm}$ & $44,57 \mathrm{ccm}$
\end{tabular}

Als Endwert $A \infty$ für eine $0,5 \mathrm{n}$ Säure wurde $44,57 \mathrm{ccm}$ gewählt; also $A \infty-A_{0}=24,37 \mathrm{ccm}$. Da bei allen Messungen dieselbe Menge Methylazetat in $100 \mathrm{ccm}$ Losung vorhanden war, ist dieser Wert für alle Messungen derselbe. Wir erhalten also für Anfangs- und Endwert der belden MeBreiken folgende Zahlen:

Tabelle ll.

\begin{tabular}{|c|c|c|}
\hline $25,0^{\circ}$ & $0.05 \mathrm{n} \mathrm{HCl}$ & $0,10 \mathrm{n} \mathrm{HCl}$ \\
\hline $\begin{array}{l}\text { Anfangswert: } A_{0} \\
\text { Endwert: } \quad A \infty \infty\end{array}$ & $\begin{array}{r}2,02 \mathrm{ccm} \\
26,39 \mathrm{ccth}\end{array}$ & $\begin{array}{r}4,04 \mathrm{ccm} \\
28,41 \mathrm{ccm}\end{array}$ \\
\hline
\end{tabular}

Die Zwischerwerte $A_{t}$ muBten je nach dem Gelatine-Gehalt um einen ihrer Azidität (siehe Seite 81) entsprechenden Betrag gekürzt werden. Diese. Korrektion beträgt für $2 \mathrm{ccm}$ bei einem Gehalt von z. B. 0,$1 ; 0,2 ; 1 ; 3 ; 6$ Proz. Gelatine 0,$01 ; 0,01 ; 0,07 ; 0,28 ; 0,42$ ccm 0,05 nLauge. Im allgemeinen wurden für jede Gelatine- und Salzsăurekonzentration mehrere voneinander unabhängige in besonderen Kölbchen angesetzte Versúchsreihen durchgeführt. Die Berechnung der Geschwindigkeitskonstanten $R$ gesehah nach der bekannten Formel:

$$
R=\frac{\log \left(A \infty-A_{0}\right)-\log \left(A \infty-A_{t}\right)}{t}
$$

Die ersten und letzten Werte für $R$ blieben wie üblich wegen Störung des Reaktionsverlaufes unberücksichtigt. (Ostwald-Luther, loc. cit. 527.) Als Beispiel einer Messung sei folgende angeführt.

\begin{tabular}{|c|c|c|c|}
\hline Minuten & $0,0 \sin ^{\mathrm{N}}$ & $\begin{array}{l}\mathrm{H} \\
\text { korr. }\end{array}$ & R.10 \\
\hline $\begin{array}{c}0 \\
1487 \\
2195 \\
2899 \\
4367 \\
5004 \\
5912 \\
6432 \\
7417\end{array}$ & $\begin{array}{r}\overline{8,56} \\
10,95 \\
12,99 \\
16,67 . \\
17,88 \\
19,33 \\
19,96 \\
21,16\end{array}$ & $\begin{array}{c}{[2,02]} \\
8,42 \\
10,81 \\
12,85 \\
16,53 \\
17,69 \\
19,19 \\
19,82 \\
21,02\end{array}$ & $\begin{array}{l}0, \overline{899} \\
0,886 \\
0,881 \\
0,900 \\
0,894 \\
0,896 \\
0,885 \\
0,886\end{array}$ \\
\hline & & & Mittel 0,890 \\
\hline
\end{tabular}


Von einer mit der Zeit forischreitenden Zersetzung der Gelatine -- etwa einer hydroiytischen Spaltung - begünstigt durch die Anwesenheit der Säure, die sich in einer Veränderung der Konstante hätte ăußern müssen, war nie etwas zi bemerken. Auf die Bestän- digkeit von Rinderalbumin gegen verdünnte Salurer haben schon $\mathrm{M}$ a nabe und $M$ atuja ${ }^{9}$ ) hingewiesen.

Die Ergebnisse der Versuche mit $0,05 \mathrm{n} \mathrm{HCl}$ sind in folgender.Tabelle zusammengesteilt.

Tabelle IV.

$25,0^{\circ}$. 0,05 n Salzsảure.

\begin{tabular}{c|c|c|c|c|c}
\hline $\begin{array}{c}\text { g Gelatine } \\
\text { im Liter }\end{array}$ & $\begin{array}{c}\text { Geschwindigkeitskonstante } \\
\text { R .104 }\end{array}$ & $\begin{array}{c}\text { Im Mitte } \\
\text { Rgei. }\end{array}$ & Rbar. & bget. & bher. \\
\hline 0 & 1,$442 ; 1,448 ; 1,448$ & 1,446 & 1,446 & 0,0500 & 0,0500 \\
1 & 1,$423 ; 1,423$ & 1,423 & 1,418 & 0,0492 & 0,0490 \\
2 & 1,$381 ; 1,395 ; 1,402$ & 1,393 & 1,389 & 0,0482 & 0,0480 \\
5 & 1,$318 ; 1,313 ; 1,315$ & 1,315 & 1,305 & 0,0452 & 0,0451 \\
10 & 1,$182 ; 1,185 ; 1,185 ; 1,83$ & 1,184 & 1,164 & 0,0410 & 0,0403 \\
20 & 0,$884 ; 0,890 ; 0,884 ; 0,883$ & 0,886 & 0,883 & 0,0306 & 0,0305 \\
30 & 0,$595 ; 0,600$ & 0,598 & 0,607 & 0,0207 & 0,0210 \\
40 & 0,$329 ; 0,337 ; 0,348$ & 0,338 & 0,344 & 0,0117 & 0,0119 \\
50 & 0,$158 ; 0,130 ; 0,140 ; 0,146 ; 0,146$ & 0,144 & 0,136 & 0,0056 & 0,0047 \\
60 & 0,$052 ; 0,052$ & 0,052 & 0,052 & 0,0018 & 0,0018
\end{tabular}

Aus den Zahlen und Fig. 1 ergibt sich, daB die $\mathbf{R}$-werte mit steigendem Gelatinegehalt zunächst proportional und etwa von $35 . \mathrm{g}$ Gelatine an immer weniger stark abnehmen.

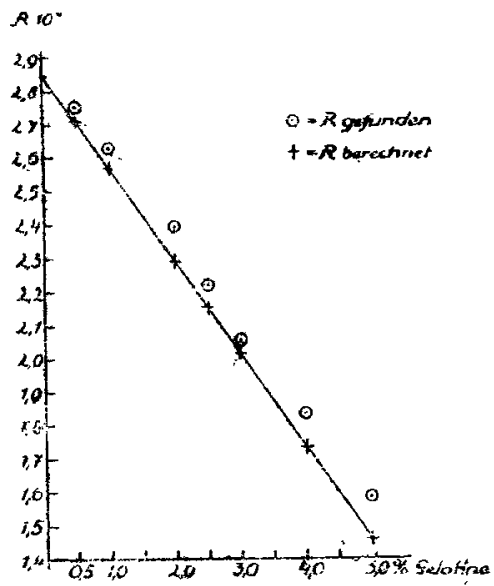

Fig: 1

Geschwindigkeitskonstante der Esterverseifung durch $0,05 \mathrm{n} \mathrm{HCl}$ bel Gegenwart von Geiatine

Da $R$ als MaB der Ceschwindigkeit der Esterverseifung proportional der Konzentration $b$ der freien Wasserstoffionen ist, so können wir diese Konzentration aus den $R$-werten berechnen nach der Gleichung $b=R c$, wo $c$ eine Konstante bedeutet. Bei dem Versuch mit 0,05 n Salzsäure ohne Gelatinezusatz erhalten wir $R=1,446.10^{-4}$. Nehmen wir an, daf die $0,05 \mathrm{n}$. Salzsäure vollständig in lonen zerfallen ist ${ }^{10}$, so wird $c=\frac{0,05}{1,446,10^{-4}}=345,8$.
Danach ergibt sich für die Lösung mit $0,05 \mathrm{n} \mathrm{HCl}$ wenn wir mit $\mathbf{m}$ die $g$ Gelatine im Liter bezeichnen, für die Wasserstoffionenkonzentration die in Tabelle IV unter $b_{\text {gef. angegebenen Werte. }}$

Dal Eiweibstoffe befahingt sind Wasserstoffionen in dieser Art zu binden, ist lange bekannt (siehe z. B. Höber, loc cit. 141, 173, $\mathrm{Pauli}$, loc, cit. 45) und wird fast allgemein datauf zurïckgefülirt, dab die eleltrisch amphoteren EiweiBstoffe in Gegenwart von Süuren wie schwache Basen reagieren, mit diesen also salzähnliche, leicht bydrolysierende Verbindungen eingehen, etwa vom Typus des Chlorammoniums oder des Aluminiumchlorids, indem sich die Salzsåure an ein oder mehrere $\mathrm{N} \mathrm{H}_{\mathrm{z}}$ - Gruppen anlagert. Nehmen: wh zunäcbst einmal nur der Einfachheit halber an, dab nur eine $\mathrm{N} \mathrm{H}_{\mathrm{g}^{2}}$ Gruppe in Reaktion träte; so liebe sich folgendes Reaktionsschema autstellen:

[Ge] $\mathrm{NH}_{2}+\mathrm{HCl}=$ [Ge] $\mathrm{NH}_{5} \mathrm{Cl}$,

Gelatinechlorid

9) Manabe u. Matula, Biochem. Zeitschr. 52 , 391 (1913).

19) Richtiger wate es, den Dissoziationsgat éter Saizsäure mit in Reelunung za. setzen; doch ist es schwierig, hicririr den richtigen Went za ermizteln. So finde: $z$. B. Ostwald 363,4 bei $25^{\circ}$; Green 397 bei $24,90^{\circ} \cdot \mathrm{Br}$ ray und $\mathrm{Hunt} 328,4$. bei $25^{\circ}$; für $\Lambda{ }_{20}^{\mathrm{HCl}}$ (sielie A begg, Handbuch 1913, iV, 139 ; Noch grißere Unsicherheit herrscht über den Wert yọ

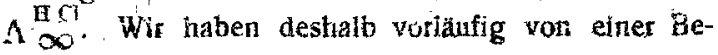
ricksichtigung des Dissoziationsgrades abgesehen, um so mehr. als aach $f$ nicht streng proportiotal der Waszerstoffionenkonzentration ist. (Slehe Lund én, Zeitschr. t. prysik. Chem. 49,196 (1904); 54,542 (1906.) 
wa [Gel den bei der Reaktion unbeeinfiußten Gelatinekomplex bezeichnen soll. In wässeriger Lösung würde sich dann ein Gleichgewicht einstellen :

[Ge] $\mathrm{NH}_{3} \mathrm{Cl}+\mathrm{H}_{2} \mathrm{O} \rightleftarrows[\mathrm{Ge}] \mathrm{NH}_{3} \mathrm{OH}+\mathrm{HCl}$ (1) oder in Form einer lonengleichung:

$$
\begin{gathered}
{[\mathrm{Ge}] \mathrm{NH}_{3}++\mathrm{Cl}^{-}+\mathrm{H}_{2} \mathrm{O} \rightleftarrows\left[\mathrm{Gel} \mathrm{NH}_{3} \mathrm{OH}\right.} \\
+\mathrm{H}+\mathrm{Cl}^{-}
\end{gathered}
$$

Wie Manabe und J. Matula (loc. cit. 392) nachgewiesen haben, ist das Gelatinechlorid im Gegensatz zum Chlorid des Albumins praktisch vollkommen dissoziiert, so dah wir, wenn wir auch die Dissoziation der Salzsäure als praktisch vollständig annehmen, sctireiben können:

[Ge] $\mathrm{NH}_{3}++\mathrm{H}_{2} \mathrm{O} \rightleftarrows[\mathrm{Ge}] \mathrm{NH}_{3} \mathrm{OH}+\mathrm{H}+$ entsprechend beispielsweise der Geleichung $\mathrm{NH}_{4}++\mathrm{H}_{2} \mathrm{O} \rightleftarrows \mathrm{NH}_{4} \mathrm{OH}+\mathrm{H}+$.

Hiernach bilden sich bei der Einwirkung von Wasserstoffionen auf Gelatine Ionen der Gelatine, ebenso wie sich bei der Einwirkung von Wasserstoffionen auf Ammoniumhydroxyd Ammoniumionen bilden. Nun enthält aber, wie die anderen Eiweibstoffe, so auch die Gelatine zweifellos nicht eine sondern mehrere Amidogruppen; ferner kommen auch noch Stellen der Peptidbindung für die Salzsäureanlagerung in Betracht. Von L. Blasel und J. Matulali) ist in der Tat gezeigt worden, dab auch das Desaminoglutin, ein durch Einwirkung von salpetriger Säure auf Gelatine erhaltenes Produkt, welches also aller Voraussicht nach keine endständigen $\mathrm{NH}_{2}$-Gruppen mehr besitzt, Wasserstoffionen $\mathrm{zu}$ binden vermag. Perner haben Pau!i und Sven $O$ dé $^{12}$ ) berechnet, da unter Berücksichtigung der Konzentration der freien Wasserstoff- und Chlorfonen aus Lèitfähigkeitsmessungen von Albumin-Salzsãuregemischen (Säuregehalt $0,075 n-0,02 n$ ) sich eine zunehmende Beweglichkeit der Proteinionen mit steigendem Säurezusatz ergibt, und daraus auf eine zunehmende Ladung der lonen, also eine zunehmende Wertigkeit, geschlossen. Nach Pauli's Vorstellung findet nur in ganz niedriger Säurekonzentration die Bildung einwertiger Proteinionen statt, d. h. nur hier reagiert das Proteinion nach obigem Schema als einwertige Base; in höherer Konzentration hingegen werden mehrere Amidogruppen zur Salzbildung herangezogen. Proteine haben nicht e in e sondern mehrere Dissoziationskonstanten von verschiedener schnell abnehmender Größe. Schon

11) L. Blasel u. J. Matula, Biochem. Zeitschr. 38, 417 (1914.)

12) Pauli u. Sven Oden, Anzeig. d. Akad. d. Wissenschaften Wien, Nr. 24 (1913.) bei zweiwertigen Săuren und Basen werden die Verhältnisse recht verwickelt und man kann wohl sagen, daf uber die verschiedenen Dissoziationskonstanten mehrwertiger Basen und Säuren bisher wenig Sicheres bekannt ist. In den meisten Fallen hat man unter dem Gesichtspunkt der Stufendissoziation derartige Elektrolyte so behandelt, als ob sich zunächst und vornehmlich einwertige Ionen in wässeriger Lösung bildeten. Diese Vorstellung wollen auch wir zunächst unserer Betrachtung zurgrunde legen und demgemäB prüfen, ob unsere Messungen sich nach obigem Reaktionsschema deuten lassen.

Für das Gleichgewicht säurehaltiger salzfreier Ampholytlösungen hat Sörensen in seinen klassischen Proteinstudien ${ }^{13}$ ) eine ausführliche Fornel gegeben.

$y=-\frac{a-s+\frac{k_{w} \beta^{\prime}}{k_{b} \alpha}}{2}+\sqrt{\frac{a-s+\frac{k^{w} \beta^{\prime}}{k_{b} \alpha}}{4}+s \frac{k_{w} \beta^{\prime}}{k_{b} \alpha}}(3)$

Darin bedeutet y die Konzentration der vom Ampholyten nicht gebundenen Săure; da wir annehmen, die freie Säure sei ganz in lonen zerfallen, so wird für uns $\mathbf{y}=\mathbf{b}$; a die Aequivalentkonzentration des Ampholyten; nehmen wir an, das Glutin reagiere als einsăurige Base, so wird $a=\frac{m}{M}$, wenn $m$ die $g$ Gelatine im Liter bedeuten und mit $M$ das Molekulargewicht der Gelatine bezeichnet wird; s die Aequivalentkonzentration der zugesetzten Säure, die wir mit $n$ bezeichneten; $k_{w}$ ist das lonenprodukt des Wassers (bei $25^{\circ}=1,1.10^{-14}$ ); $\mathbf{k}_{b}$ die basische Dissoziationskonstante des Ampholyten; darnach wird $\frac{k_{w}}{k_{b}}=K$, die $\mathrm{Hy}$ drolysenkonstante. $\alpha$ und $\beta^{\prime}$ bedeuten die Dissoziationsgrade der Säure und des Ampholytsalzes. Bei seinen Berechnungen setzt Sörensen den Dissoziationsgrad des Ampholytsalzes gleich dem von $\mathrm{NH}_{4} \mathrm{Cl}$ in derselben Konzentration. Ueber den Dissoziationsgrad des $\mathrm{NH}_{4} \mathrm{Cl}$ gilt aber das Seite 83 für Salzsäure gesagte ${ }^{14}$ ). Wir haben deshalb und auch aus andern Gründen ${ }^{15}$ ) geglaubt, $\alpha=\beta^{\prime}$ setzen

19) Zeitschr. f. physiol. Chem, 103, 134 (1918).

14) Siehe A begg, Handbuch 1907, $\mathrm{III}_{8}, 259$. Mit dea meist benutzten Zahlen wird der Dissoziationsgrad des $\mathrm{NH}_{6} \mathrm{Cl}$ kleiner als der der Salzsaure derselben Konzentration, was wohl unwahrscheinlich ist.

15, Wie Ma nabe u. Matula, Biochem. Zeitschr. 52, 381, 392 (1913) gezeigt haben, werden bei der Einwirkung verduinntếf Salzsäure auf Rinderalbumin 
zu dürfen, schon um die Rechnung zu vereinfachen. Dann geht Gleichung (3) über in * $b=-\frac{1}{2}\left(\frac{m}{M}-n+K\right)+\sqrt{\frac{1}{4}\left(\frac{m}{M}-n+K\right)+K_{n}{ }^{16} \cdot(4)}$

Gleichung (4) erhält man natürlich auch unter den oben gemarhten Voraussetzungen unmittelbar aus der Gleichung für die Hydrolysenkonstante

$$
K=\frac{b\left[\frac{m}{M}-(n-b)\right]}{n-b}=\frac{b m}{M(n-b)}-b
$$

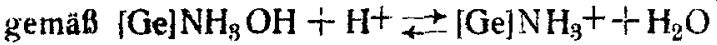

$$
\frac{\mathbf{m}}{\mathrm{M}}-(\mathbf{n}-\mathbf{b}) \quad \mathrm{b} \quad \mathrm{n}-\mathrm{b}
$$

Nach Formel (4) ergibt sich aus den oben angegebenen (b proportionalen) $R_{\text {get }}$ - Werten durch Ausgleichrechnung $M=1014^{17}$ ) und $K=4,139$ $.10^{-4}$. Hieraus ergibt sich für die basische Dissoziationskonstante

$$
k_{h}=\frac{k_{a}}{K}=\frac{1,1 \cdot 10^{-1}}{4,14 \cdot 10^{-4}}=2,7 \cdot 10^{-11}
$$

Für Alanylglyzin, Glyzylglyzin und Leuzylglyzin (wohl die enzigen bisher untersuchten amphoteren Stoffe mit Peptidbindung) beträgt, $\mathrm{k}^{b} 2,1$ v. 2,0 u. $3,1 \cdot 10^{-11}$ (Lundén, s. u.), Hiermit sind die unter $R_{\text {ber, }}$ und $b_{\text {bey, }}$ angegebenen Werte berechnet. Wie man siehr, stimmen die gefundenen und berechneten Werte gut überein. Man kann also nach Gleichung (4) die unter der Annahme abgeleitet ist, Gelatine reagiere wie eine schwach einsäurige Base, die Wasserstoffonenkonzentration in Gleichgewicht GlutinSalzsarure berechnen.

Berechnet man mit denselben Werten für $M$ und $K$ und dem Faktor $c=\frac{0,1}{2,850 \cdot 10^{-4}}=350,9$ (s. oben) $R$ und $b$ für die Konzentration 0,1 der Salzsäure, so ethalt man die in Tabelle $V$ und Fig. 2 angegebenen Werte.

\begin{tabular}{|c|c|c|c|c|c|}
\hline $\begin{array}{l}\text { g Oetatine } \\
\text { in Ltter }\end{array}$ & $\begin{array}{l}\text { Geschwindigkeitskonstante } \\
\text { R.104 }\end{array}$ & Im Mittel & Rber. & $b_{\text {geat. }}$. & Bbar. \\
\hline & $x+1=10$ & & & & \\
\hline $\begin{array}{r}0 \\
5 \\
10 \\
20 \\
25 \\
30 \\
40 \\
50\end{array}$ & $\begin{array}{ll}2,843 ; & 2,857 \\
2,747 ; & 2,760 \\
2,623 ; & 2,636 \\
2,395 ; & 2,400 \\
2,220 & \\
2,054 & \\
1,836 & \\
1,589 & \end{array}$ & $\begin{array}{l}2,850 \\
2,754 \\
2,630 \\
2,398 \\
2,220 \\
2,054 \\
1,836 \\
1,589\end{array}$ & $\begin{array}{l}2,850 \\
2,710 \\
2,570 \\
2,291 \\
2,451 \\
2,012 \\
1,733 \\
1,456\end{array}$ & $\begin{array}{l}0,1000 \\
0,0966 \\
0,0923 \\
0,0842 \\
0,0779 \\
0,0721 \\
0,0644 \\
0,0558\end{array}$ & $\begin{array}{l}0,1000 \\
0,0951 \\
0,0902 \\
0,0804 \\
0,0755 \\
0,0706 \\
0,0608 \\
0,0511\end{array}$ \\
\hline
\end{tabular}

Tabelle V.

$25,0^{\circ}$. $0,10 \mathrm{n}$ Salzsäure.

Die gefundenen Werte sind durchweg etwas gröBer als die berechneten. Worauf dies zurückzuführen ist, läBt sich schwer sagen. Sicher werden hierbei die in der Rechnung

Chlorionen in steigendem Maße gebunden. Bein salzsauren Clutin dagegen ist die Dissoziation bedeutend stärker.

Nach Arrhenius ist in einem Gemisch zwerer starker Elektrolyte mit einem gemeinsamen lon der Dissoziationsgrad jedes Elektrolyten durch die Konzentration des gemeinsamen Ions bestimmt.

16) Diese Gleichung haben wir anch in der when angefuhrten vorläufigen Mittcilung unserer Berechnung zu Grunde gelegt:

17) Einen annahernden Wert von $M$ erhals man natürlich auch aus Fig. 1, wenn man den oberen geradlinig verlaufenden Teil der Kurve bis zum Schnittpunkt mit der Abszissenachse verlangert. Bej 5,1 Proz. Gelatine würde $\mathrm{R}$ in Gegenwart von $0,05 \mathrm{n} \mathrm{HCl}$ gleich $O$, wenn keine Hydrolyse einträte, $d . h$. bei diesem Punkte waretı alle $\mathrm{H}$-Ionen verschwunden. $1 \mathrm{Mol}$. $\mathrm{HCl}$ wtirde also rund $1020 \mathrm{~g}$ Gelatine binden.

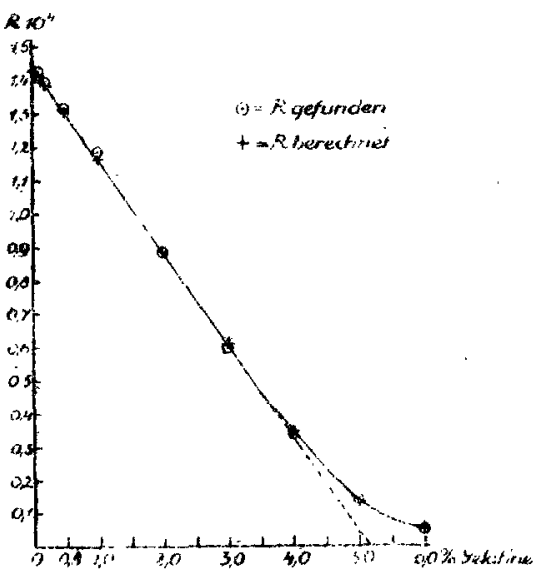

Fig. 2

Geschwindigkeitskonstante der Esterverseifung durch $0,10 \cap \mathrm{HCl}$ bel Gegemwart von Gelatine. 
nicht mit berïcksichtigten und wie ohen auseirandergesetz schwer mit Sicherheit zu schatizenden Dissoziationsgrade der Elektrolyte und wahrend der Verseifung die sog. Neutralsalzwirktug eine Rolle spielen. Würden, woran wan zunächst denken könnte, in Gegenwart de: stärkeren Salzsäure noch neue $\mathrm{NH}_{2}$ Gruppen mit Salzbilutung herangezogen, so ist im Gegenteil eine Verminderung der H-Lonen, also eine Abweichung in der entgegengesetalen Richtung zu erwarten. Aus den $\mathrm{R}_{\text {pat.-Werten er- }}$ gibt sich schatzungsweise $M=1090 . \quad \mathrm{Da}$ die luftircckene Gelatine noch 17,25 Droz. Wasser enthait, also 82,75 Proz trockne Gelatine, ist noch eine Korrektion anzubringen. Nach Formel (5) ergiht sich für zwe: verschiedere salzsaure Lösungen lifttrockener Gelatine

$$
K=\frac{m_{1} b_{1}}{M\left(\mathbf{n}-b_{1}\right)}-b_{1}=\frac{m_{2} b_{2}}{M\left(\mathbf{a}-b_{2}\right)}-b_{2},
$$

darass folgt

$$
M=\frac{1}{b_{2}-b_{1}}\left[\frac{b_{2} m_{2}}{n-b_{2}}-\frac{b_{1} m_{1}}{n-b_{1}}\right]
$$

för 82,75 Proz. Gelatine hat man $m_{1}$ und $m_{2}$ noch mit dem Paktor $f=\frac{82.75}{100}=0.8275 \mathrm{zu}$ multiplizieren, also auch $M=1014$; folglich $M_{\mathrm{H}_{\mathrm{z}} \text { ○ fret }}=839$. Die Hydrolysenkoustante $K=4,139 \cdot 10^{-}$andert sich nicht, da in Gleichung (5) sowohl $m$ wie $M$ mit $f$ zu multiplizieren sind, Diese Verhältnisse ergeben sich auch ohne weiteres aus sen Piguren 1 und 2 , Unter Berücksichtigung der Korrektion für das Wasser rücken alle Werte anf der $\mathrm{x}$-Achse proportionai nach links. Dadurch tritt eine Parallelverschiebung der ganzen Kurve und tamit auch eine Verschicbung des Schnittpunktes ibres geraderi Teiles mit der Abszissenachse, welche $M$ bestimmt, nach links ein. Krummung und Rtchtung der Kurve, von denen $\mathrm{K}$ abhängt, bleiben unverändert.

Der K-We:t karn keinen groben Anspruch auf Genaulgkeit machen, da et sich natürlich nur aus dem verhältnismälitg kleinen Krümmuingsturck der Kurve ergib:

Wir haben sodann gepraft, ob auch die Messungen yon $P_{\text {a }}$ uli und seinen Mitabibeifern ${ }^{18}$ ) sich nach Gleichung (4) berechnen-lassen. Wir haben. zu difespm Zweck nicht den langwierigen Weg Ger Auggleicirechnurg eingesthiagen, sondern folgender: Srhreibt man oleichung (5) in der

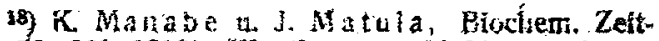
schr. 52; 369 (1913); Wo. Pali u. M. Hi: aghteld 62, 245 (1914); L. Blaselu. J. Matula, 58, 417 (1914).
Porm: $b=\frac{1}{M} \cdot \frac{m \cdot b}{n-b}-K$, so drückt diese aus; $d a b$ e eine lineare Funkrion von $\frac{m b}{a-b}$ ist. Trägt man also zusammengehörige Werte von $b$ und $\frac{a b}{n-b}$ in ein Koordinatensystem ein, so mub man, wenn dic Messungen sich nach Formel (4) ausdrücken lassen, eine gerade Linie erhalten. Wie Fig. 3 zeigt, ist das im groben und ganien der Fall. Die Werte in der Nahe des Nullpunktes der Geraden konnten nicht alle eingezeichnet werten. Auch der letzte Wert in der Tabelle llb von $L$. Blasel und J. Matula, loc. cit., ist in der Pigur nicht berücksichtigt. Ër liegt nicht in der Fortsetzung der Geraden sondern höher ${ }^{19}$ ), der nächst höhere ebenfalls nicht mit aufgenomme Wert aus der Kurvenangabe (Pauli u. Hirsch. feid, loc. cit., 256) entnommen, liegt aber wieder auf der Verlängerung der Geraden.

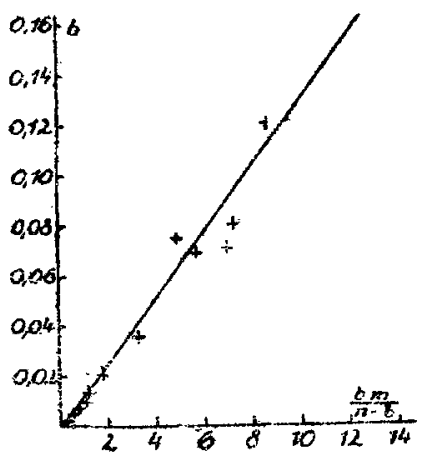

Fig. 3

Glutin (berechnet nach Zahien von $\mathrm{Pa}$ a li, Matula, Blasel, Manabe, Hirschfeld; Biochem. Zeitschrift 52,$369 ; 58,417 ; 62,245$ ).

Aus den Werten für i Proz. Glutin und $2,0 \cdot 10^{-2}$ und $5,0 \cdot 10^{-3}$ haben wir $M=881,4$, $K=1,473 \cdot 10^{-4}, k_{b}=\frac{0,6 \cdot 10^{-14}}{1,47 \cdot 10^{-4}}=4,08 \cdot 10^{-11}$ berechnet. (DaB dieser Wert für Zimmertemperatut gröber tst als der von uns bel $25^{\circ}$ ermittelte liegt wohl auch daran, dab in den Rechnungen mit $P_{a} u i^{\prime}$ 's usw. Zahlen bei $b$ der Dissoziationsgrad der Salzsäure berücksichuigt ist.)

In der folgenien Tabelle VI sind die gefundenen und die nach Formel (4) berectneten

in) Dieses kơnote man darauf zuruckfihrci. daA bei der hotien $\mathrm{H}^{+}$-Konzentration mehr Amidogruppen slich an der Sazblldung beteiligen. Fir djesen Punkt berechnet sich $M$ zy yd 500 . 
Werte zusammengestellt. Bei dieser Rechnung haben wir wie gesagt für n nicht die Konzentration der gessmten Săure sondern nur den nach den Angaben Pauli's und seiner Mitarbeiter berechneten dissozilerten Anteil derselben zu Grunde gelegt. Entsprechend dem Verlaui der Geraden ist auch hier die Uebereiristimmung befriedigend, wonn man berïcksichtigt, daB die Messungen - wohl bei wechselnder Zinmertemperatur ${ }^{20}$ ) ausgaeführt manchmal auch unter sich betrachtlich $a b$ weichen (siehe die beiden Versuche mit 1 Proz. Gelatine und $5 \cdot 10^{-5} \mathrm{n}$ Salzsäure):

\section{Tabelle VI.}

\begin{tabular}{|c|c|c|}
\hline $\mathrm{AF}^{3} \mathrm{Cl}$ & gef. & ber. \\
\hline \multicolumn{3}{|c|}{1 Proz. Gelatine } \\
\hline $\begin{array}{l}5,0,10=2 \\
2,0 \\
2,0 \\
1,5 \\
1,0 \\
1,0 \\
7,0.10-3 \\
5,0 \\
5,0 \\
3,0\end{array}$ & $\begin{array}{l}3,28 \cdot 10-2 \\
8,02 \cdot 10-3 \\
6,93 \\
3,60 \\
9,78 \cdot 10-4 \\
8,72 \\
1,58 \\
1,07 \\
1,57 \\
1,84,10-5\end{array}$ & $\begin{array}{l}3,57 \cdot 10^{-2} \\
8,02 \cdot 10^{-3} \\
8,02 \\
3,57 \\
5,88 \cdot 10^{-4} \\
5,88 \\
2,05 \\
1,07 \\
1,07 \\
5,00 \cdot 10^{-5}\end{array}$ \\
\hline \multicolumn{3}{|c|}{ 0,75 Proz. Gelatine } \\
\hline $\begin{array}{l}5,0.10^{-3} \\
2,0 \\
1,0\end{array}$ & $\begin{array}{l}3,15 \cdot 10-2 \\
7,56 \cdot 10-8 \\
1,34\end{array}$ & $\begin{array}{r}3,85 \cdot 10^{-2} \\
10,77 \cdot 10^{-3} \\
1,80 \cdot 10^{-3}\end{array}$ \\
\hline \multicolumn{3}{|c|}{0,5 Proz. Gelatine } \\
\hline $\begin{array}{l}2,0 \cdot 10^{-2} \\
1,0 \\
5,0 \cdot 10-3 \\
2,5\end{array}$ & $\begin{array}{l}1,21.10-2 \\
3,66.10-8 \\
6,92.10-4 \\
1,42\end{array}$ & $\begin{array}{l}1,36 \cdot 10-2 \\
4,18 \cdot 10-8 \\
4,96 \cdot 10-4 \\
1,04\end{array}$ \\
\hline \multicolumn{3}{|c|}{0,25 Proz. Gelatine } \\
\hline $\begin{array}{l}2,0.10-2 \\
1,0 \\
5,0.10-8 \\
2,5 \\
1,25\end{array}$ & $\begin{array}{l}1,59 \cdot 10^{-2} \\
7,12.10^{-2} \\
2,03 \\
4,80.10^{-2} \\
13,77.10^{-5}\end{array}$ & $\begin{array}{l}1,63 \cdot 10^{-2} \\
6,89.10^{-2} \\
2,21 \\
3,91.10^{-1} \\
9,81.10^{-6}\end{array}$ \\
\hline
\end{tabular}

Es war von Interesse, welche Resultate mit uriserer Berechnungsweise die Messungen von Manabe und $J$. Matula ${ }^{3}$ ) sowie von $L$.

20) Die Hydrolyse der Salze sehr schwacher Basen mit sehr starken Säuren nimmt allerdings nur. unbedeuterid mit der Temperatur z"n. (Lundén. Afflnitätsmessungen, Stuttgatt 1908,5 . 69. Walker, Einf. is d pins. Chemie, 2. Aufl, Braunschweig 1914, S. 407).

2) Manabe u. J. Matula, loc. cit. 52, 394
Blasel und .. Matula ${ }^{25}$; am Desaminoglutin ergeben wärden.

Desaminoglutin (Manabe und J. Matula): 8 Wochen dialysiert, $C_{H}$ einer 0,15 prozentigen Lösung $3,14 \cdot 10^{-7}$. Zusammengehörige Werte von $\frac{m n}{n-m}$ und $b$ liegen nicht auf einer geraden Linie. $M=224,2$ (aus Versuch 1 und 3 , loc. cit. 394).

Desaminoglutin (L. Blasel und J. Matula): 2 Wochen dialysièr, $C_{\mathrm{B}}$ einer 0,75 prozentigen L.ösung $1,79.10^{-5}$. Zusammiengehơrige Werte von $\frac{m n}{n-b}$ und $b$ liegen auf einer geraden Linie (s. Fig. 4) $M=918,3$ (aus Versuch.1 und 5, loc. cit. 432$) \mathrm{K}=9,4.10^{-4}, \mathrm{k}_{\mathrm{b}}=6,4.10^{-12}$. Trutz. Verlust der endständigen $\mathrm{NH}_{2}$-Grippen hat sich das "Molekulargewicht" nicht wesentlich geändert. (Nach den Angaben von Wo. Pauli ${ }^{20}$ ) sollte man etwa 820 erwarten), ein Resultat, das wiederum für $M$-Glntis $=839$ spricht, denn sicher neigः das Desaminoglutin noch mehr zur Bildung nur einwertiger Ionen als das Glutin:

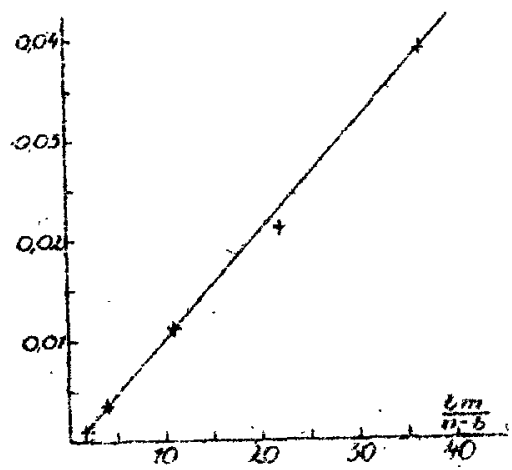

Fig. 4

Desaminoglutin (berechnet nach Zahlen von Blase! und Mat ula; Biociem. Zeitschr. 58, 423).

Auf einem andern Wege ist das Gleichgewicht Gelatine-Salzsaure von H. R. Procte ${ }^{24}$ ) untersucht worden. $\mathrm{Er}$ lieb Gelatine in verdünnter Salzsäure bis zum Eintritt des. Gleich-

26) L. B lasel u. J. Matula, toc. cit. $58 ; 417$.

28) Wo Paill, Kollididchemie der Eiweid. körper, 70 .

as) H. R. Procter, Kolloidchem. Bein. 2. 243 (1911); Journ. Chem. Soc. 105, 313 (1914). British Assoc. for the Advancement of Science, First Rep. on colloid Chemistry and its industrial applications 1917, 5 . Procter gibt hier eine zusammenfassende Darstellung des Problems von gerbereitechnischen Standpinkt.) 
gewichts quellen, setzte die gequollenen abgetrockneten Stücke dainn der Einvirking von festem Kochsalz aus, wodurch diese unier $\mathrm{Ab}$ gabe der überschüssigen salzsauren lösung bis zur hornartıgen Konsistenz einschrumpten and bestimmte durch Titration die Säuremenge, welche gebunden bleibt. Unter der Annahme, daß das Glutin eine bei niedriger Saurekonztntration zh vernachlässigende $z \mathrm{~W}$ eite basische Valenz ${ }^{25}$, hat, fand er aus der so ermittelten Hydrolysenkurve ein Verbindungsgewicht ron 839. I. A. Wils on ${ }^{26}$ ) ist der Ansicht, dab die zweite Valenz von beginnender Zersetzung herrühre oder durch Analysenfehler vorgetäuscht sei und berchnete mit Procter's Daten 768. In ahnlicher Weise untersuchte V. Kabelka ${ }^{27}$ ) das verwickelte Gleichgewicht Salzsäure - Hautpulver und berechnet, daf die bei niedriger Konzentration der Säure vermutlich entstehende Verbindung auf 1 Grammäquivalent Salzsäure $977 \mathrm{~g}$ Hautkollagen enthält.

M. B errar ${ }^{28}$ ) fand, dab Glutin berm Fäilen mit einem Gemisch von Pikrinsăure und Quecksilberjodici-Jodkalium unablängigs von den Mengenverhältnissen auf $823 \mathrm{~g}$ stcis $1 \mathrm{Mol} \mathrm{HCl}$ aufnimmt. A. Guttenberg ${ }^{29}$ ) mak die Salzsäurebindung des Glutins mit Hille der $\mathrm{G}$ ii $\mathrm{n}_{2}$ berg'schen (Phlorugluzin -Yaniuin) Reaktion. Aus seinen Zablen ergibt sich, daB $1 \mathrm{Mul} \mathrm{HCl}$ $788 \mathrm{~g}$ Glutin äquivalent ist. Von Procter (loc. cil.) ist auch schon darauf hingewiesen worden, dab der von $\mathrm{h} h \mathrm{~m}$ und uns gefundene Wert 834 der Formel $\mathrm{C}_{65} \mathrm{H}_{57} \mathrm{O}_{13} \mathrm{~N}_{11}$ eutspricht, wriche den analytischen Resultaten von Schütrenbergcr und Bourgeo is ebenso gut gerecht wird wit die von diesen vorgeschlagene $\mathrm{C}_{76} \mathrm{H}_{12} \mathrm{O}_{4,4} \mathrm{~N}_{24}$. Aus Siedepunktserhöhungen wässeruger Gelatinelösungen verschiedener. Konzentration erhielt $\mathrm{C} . \mathrm{Pa}$ al ${ }^{30}$ iiir das Molekulargewicht der Gelarine Werte, die 2 wischen 878 und 960 ligzen. Alles dies sicheint unserer Aisicht nach darauf hinzudeuten, dab Glutin (falls es uberhaupt cine einheilliche Substanz ist) in wässeriger lossing bei Gegen. vart von verdünnter Salzsầute und elenso in

2: Für die 1. und 2. basische Dissoziationskon. starte cigeben sich die Werte $0,5.15-11$ i. $0,6.10-1 \%$.

w) i. A.Wilson, Jomm of the American Leathe 6hemist's Association 12, 108 (1917).

8) V. Kubelka, Koll.-Zeitscirr. 23,57 (1918).

wi) M. Berrat, Biochern. Zeitsclir. 17, 205 (1912),

4) A. Guttenberg. Münchenes med. Wochenjihrift 13, 147 (1896). (352;

4ii, C Paal, Ber. d Deutscl, chem. Ges 25, 1202 der Hitze in eine Verbindung vom Aequivalentgewicht, vielleicht auch vom Molekulargewicht 800 ... 900 übergeht. Gegen das Molekular. gewicht sprechen auber den bereits oben auseinandergesetzten Gründen noch zahlreiche andere.

Zwar sind nach $\mathrm{G}$. B re di ${ }^{31}$ ) das Hydrazin, das Guanidin und der Harnstoff, obwohl sie mehrere Amidogruppen haben, ausgesprochen einsäurige Basen, und Hydrazin läßt sich scharf als einsäurige Base titrieren. Die freien Amido. gruppen der Monuchlorhydride dieser Verbindungen zeigen also nur noch verschwindend geringe basische Reaktion. Aber das basische Chlorhydrid des Aethylendiamins ist bereits erheblich stärker basisch als das basische Chlorhydrid des Hydrazins, So zeigen wässerige Hydrazindichlớidiosungen fast dieselbe Leitfahigkeit wie sich fur ein Gemisch von $1 \mathrm{Mol}$ Salzsäure und $1 \mathrm{Mol}$ Hydrazinmonochlorid berechnet. Wásserige Lösungen von Aethylendiamindichlorhydrid zeigen aber ungefähr nur die Hälfte der Leitfähigkeit wie sich für ein Gemisch von I Mol Salzsäure und $1 \mathrm{Mol}$ Aethylendiaminmonochlorhydrid berechnet. Aethylendiamin läbt sich bereits scharf $z$ wcibasisch titrieren. G. Bredig zeigte ferner, daf auch" das Dichiorid des lysins, welches uns als wohldefiviertes Spaltungsprodukt des Eiweib hier besonders inieressiert, stark hydrolysiert, während das Monochlorid kaum Spuren von Hydrolyse zeigte. A. Kanitz ${ }^{32}$ ) hat dann die erste tind zweite basische Dissoziationskonsiante einiger bekannter Eiweißabbaiprodukte genauer untersucht und fand.

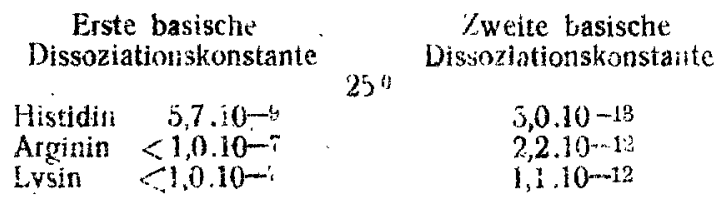

Die zweite Koristante is' also gegenúber der ersten sehr schwach.

Aus den nacher noch zu bespredienden Versuchen über die Verseifungsgeschwindig. keil von Methylazetat in Gegenwart solcher Ampholytsalze fand er beim Histidindichlorid in der Verdünnng 10 als das 100 fache des bydroiysierten Molenbruchteils $100 \mathrm{x}=31,8$; bcim Lysindichlorid in der Verdünumang 10 bzw. $20100 x=22,4$ und 30,8 ; beim Arginindinitrat 1894

ai) G. Bredig, Zeitschr. f. physik Chem 18, 315 $476(1906)$ 
in der Verdünnung $10,75100 \mathrm{x}==19,5$. Trotz. dem also die zweiten basischen Dissoziation:konstanten sehr schwach sind, macht augenscheinlich bei Säureüberschuls die Salzbildung bei der ersten Gruppe nicht halt. Wir haben nun nach Angaben von A. Kanitz und entsprechenden Messungen von J. Walker ${ }^{33}$ ) aus Harnstoff nach Formel (6) aus je zwei Messungen das Molekulargewicht in berechnet. $\checkmark$ Walker fand als Konstante der Verseifungs. geschwindigkeit C' für seine n Salzsäure 0,00315. für das Gemisch 1 Mo! Salzsäure und 1'z Mol Harnstoff 0,00228 ; für 1 Mol Salzsảure und 4 Mol Harnstoff 0,000503. A. Kanitz, loc. cit. für $0,1 \mathrm{n}$ Salzsäure $C=3,165 \cdot 10^{-1}$, für Histidindichlorid $\left(\mathrm{V}=10, \mathrm{C}=1,01.10^{-4}\right.$, für Histidinhydrochlorid $(\mathrm{V}=10) \quad \mathrm{C}=1,84.10^{\prime \prime}$ (c' zeigte schlechte Konstanz; $1,84.10^{-6}$ ist das Mittel aus den drei ersten Werten), für Lysindichlorid $(V=10) C=7,07.10^{-5}$ und fïr Lysindicblorid $(V=20) C=4,88 \cdot 10^{-6}$. Daraus ergaben sich für Formel (6) folgende Zahlen:

Tabelle VII

\begin{tabular}{|c|c|c|c|c|c|c|c|c|}
\hline & $\mathrm{m}_{1}$ & $\mathrm{~m}_{2}$ & $\mathrm{n}_{1}$ & $\mathrm{n}_{2}$ & $b_{1}$ & $b_{2}$ & $\begin{array}{c}M \text { nach } \\
\text { Formel }(6)\end{array}$ & $M$ \\
\hline $\begin{array}{l}\text { Harnstoff } \\
\text { Glyzin*) } \\
\text { Histidin. } \\
\text { Lysin } \\
\text { Glyzylglyzin *) }\end{array}$ & $\begin{array}{l}10 \\
30,024 \\
10 \\
14,6 \\
26,42\end{array}$ & $\begin{array}{l}240 \\
30,024 \\
10 \\
7,3 \\
26,42\end{array}$ & $\begin{array}{l}1 \\
0,1 \\
0,2 \\
0,2 \\
0,1\end{array}$ & $\begin{array}{l}1 \\
0,5 \\
0,1 \\
0,1 \\
0,2\end{array}$ & $\begin{array}{l}0,7238 \\
1,306.10-: 3 \\
0,0319 ! \\
0,02234 \\
0,7047.10-3\end{array}$ & $\begin{array}{l}0,1597 \\
106,42,10-3 \\
0,0005813 \\
0,01542 \\
0,0760.10-3\end{array}$ & $\begin{array}{c}59,87 \\
73,5 \\
91,01 \\
73,0 \\
137,9\end{array}$ & $\begin{array}{l}150 \\
75 \\
155 \\
146 \\
132,1\end{array}$ \\
\hline
\end{tabular}

*) S. P. L. Sörensen, Zeitschr. t. physik. Chemie 103, 151 (1918),

* K. G. Deraby, Comp. rend, du Laboratoire de Carlsberg 11, 269 (1916).

Bei Harnstoff und Glyzin ergibt sich also das theoretische Molekulargewicht, bej Lysin die Hälfte, beim Histidin mit dem 2 und 3. Stickstoffatom itn Ring ein mittierer Wert ${ }^{34}$ ). Das Glyzylglyzin reagiert also bei Gegenwart von Salzsäure nicht mit dem Peplidstickstoff. Wenn also auch das Desaminoglutin noch $\mathrm{H}$-lonen bindet, bratchen doch den Peptidsttckstoffatomen im Glutin noch keine nennenswerten basischen Eigenschaften zugeschrieben zu werden ${ }^{35}$ ).

Trotzdem sind wir schleblich der Meinung. dab, wenn auch bei der Salzsäurebindung des Glutins hauptsächich oine basische Gruppe sich beteiligt. man nicht ohne weiteres nach Formel (4) 839 als das Molekulargewicht des Glutins annehmen kann. Man tut vielmehr besser, dieser Zahl vorläufig nur den Wert eines Verbindungsnder Aequivalentgewichtes zuzuschreiben und dementsprechend unserer Hydroly senkonstanten bzw Dissoziationskonstanten - den Wert einer mittlerer oder reduzierter Konstanten in Sinne 11889 )

96, Wa 1 ker. Zeitschr. f: physik. Chem. 4, 324

34) Vielloicht ruhrt die Ahweichung aber auch von dem ungenatsen $C$. Wert her (siehe oben:.

95, Die Untersuclung synthetischer Polypeptide in dieser Richtung ist in Aigriff genommen. von Wo Pauli und M. Hirschfeld ${ }^{36}$ ). Vorn rein chemischen Standpunkte aus kann man sihon aus der Art und Zanl der Spaltungsprodukte des Glutins auf ein weil höheres Molekulargewicht schlieBen. Aus den relativen Mengen dieser Spaltungsprodukte nach $D$. D. van Sly ke ${ }^{37}$ ? und ihrem Stickstoffgehait berechnete sơ D. J. Lloyd ${ }^{38}$ ) kürzlich als niedrigstes Molekulargewicht der Gelatine angenähert 10300. Inmerhin bleibt es unserer Meinung nach sehr. beachtenswert, caB Paal's Molekulargewicht aus der Siedepunktserhöhutig praktisch mit dem Wert 839 übereinstimmt.

Ii rein wăsseriger Lösung, also ohne Salzsáurezusatz und bej gewöhnlicher Temperatur liegen natürlich ganz andere Verhältnisse vor. Nach Zähigkeit, osmotischem Druck, Diffusionsgeschwindigkeit und ultramikroskopischem Aus. sehen $x$ u schlichen, handelt es sich hier nicht um Einzelmoleküle sondern um Molekülaggregate.

Der experimentelle Teil der Arbeil wurde im Chemischen Institut der Universität Bonn ausgeführt.

ox) Wo. Pauli a. M. Hirschfeld, Biochem. Zeitschr. 62, 256 (1914).

37) D. D. va i Sly ke, Journ. Biol. Chem. 10, 15. 38) D. J. Lloy d, Biochem. fourn. 14, 147 (1920). 